\section{Human telomerase and Cajal body ribonucleoproteins share a unique specificity of Sm protein association}

\author{
Dragony Fu and Kathleen Collins ${ }^{1}$ \\ Department of Molecular and Cell Biology, University \\ of California at Berkeley, Berkeley, California 94720, USA
}

Cajal bodies are nuclear structures that host RNA modification and assembly reactions. Some RNAs transit Cajal bodies, while others must concentrate in Cajal bodies to function. Here we report that at least a subfraction of human telomerase RNA and individual resident Cajal body RNAs is associated with Sm proteins. Surprisingly, of seven $\mathrm{Sm}$ proteins assembled into a heteroheptameric ring, only a subset copurifies telomerase and Cajal body ribonucleoproteins. We show that a Cajal body RNA localization motif determines this specificity. These discoveries expand the cellular repertoire of $\mathrm{Sm}$ protein assemblies and their involvement in ribonucleoprotein localization and function.

Supplemental material is available at http://www.genesdev.org.

Received November 4, 2005; revised version accepted

December 28, 2005.

Cajal bodies are evolutionarily conserved domains present in the nuclei of a diversity of animal and plant cells (Gall 2003). Specific RNA modification and ribonucleoprotein (RNP) assembly factors are enriched in Cajal bodies, suggesting that these structures reflect the physical scaffold for biochemical coordination of steps in cellular RNP biogenesis (Cioce and Lamond 2005). Consistent with this role, Cajal body transit of RNAs correlates with processing and assembly events required for production of mature RNP (Verheggen et al. 2002; Jády et al. 2003). Many RNAs that transit Cajal bodies and proteins that assemble with these RNAs adopt a steady-state localization elsewhere.

In addition to transiting molecules, Cajal bodies also harbor components that, despite nucleoplasmic exchange, remain enriched in Cajal bodies (Gall 2003; Cioce and Lamond 2005). Resident Cajal body proteins include RNA-processing enzymes, RNP assembly chaperones, and proteins with putative structural roles. More recently, resident Cajal body RNAs have been identified, termed small Cajal body RNAs (scaRNAs), which can guide the sequence-specific modification of small nuclear RNAs (snRNAs) during their assembly into spliceosomal snRNPs (Darzacq et al. 2002; Jády et al. 2003). The snRNA modifications gained during Cajal

[Keywords: telomerase ribonucleoprotein; Smith autoantigen; Cajal bodies]

${ }^{1}$ Corresponding author.

E-MAIL kcollins@berkeley.edu; FAX (510) 643-6334.

Article published online ahead of print. Article and publication date are at http://www.genesdev.org/cgi/doi/10.1101/ gad.1390306. body transit are important for RNP biogenesis and function, as exemplified by the demonstration that U2 snRNA modification is required for its assembly into functional snRNP (Yu et al. 1998). Furthermore, snRNA modification requires the snRNA to transit Cajal bodies, because a scaRNA RNP (scaRNP) cannot modify a transcript targeted elsewhere (Jády et al. 2003). These and other studies indicate that proper nuclear compartmentalization greatly influences scaRNP function.

The RNA subunit of human telomerase can be detected in Cajal bodies (Jády et al. 2004; Zhu et al. 2004), although the functional significance of this localization is not yet clear (Pederson 2004). The human telomerase RNA (hTR) contains a box H/ACA motif shared by many scaRNAs and a large family of small nucleolar RNAs (snoRNAs) as well (Mitchell et al. 1999a; Henras et al. 2004). The H/ACA motif recruits four proteins, including the pseudouridine synthase Cbf5p/dyskerin, forming an RNP that can recognize and modify specific site(s) of a target RNA molecule (Henras et al. 2004). Although H/ACA-motif snoRNPs and scaRNPs share protein components and a common biogenesis pathway, the snoRNA family must function in nucleoli to guide modification and cleavage of ribosomal RNAs, while the scaRNA family must function in Cajal bodies to guide modification of snRNAs. Vertebrate telomerase RNA, despite its assembly with the same four H/ACA-motif-binding proteins, must function at chromosome ends and potentially at other locations as well (Blasco 2002).

RNA loops capping the H/ACA-motif stems of scaRNAs have a 4-nucleotide Cajal body localization determinant termed the CAB box (Richard et al. 2003). The same motif is found in the 3' stem-loop of hTR (Jády et al. 2004). Factors associated with scaRNAs dependent on the CAB box have remained unknown. Here, we describe our discovery of proteins associated with human telomerase and Cajal body RNPs with specificity dependent on the CAB box.

\section{Results and Discussion}

$S m B$ and $S m D 3$ are associated with human telomerase

We discovered $\mathrm{Sm}$ proteins within a list of proteins identified by mass spectrometry as being associated with affinity-purified human telomerase (data not shown). Sm and like-Sm proteins comprise an evolutionarily conserved family of Sm-fold proteins that participate broadly in determining the cellular fate of RNA (Khusial et al. 2005). These proteins are known to assemble in specific combinations to form heteroheptameric complexes with distinct functions. The prototypical Sm proteins B, D1, D2, D3, E, F, and G recognize and encircle the uridine-rich (U-rich) Sm-binding site present in most snRNAs, exploiting sequential assembly of SmD1-D2, SmE-F-G, and SmB-D3 subcomplexes to form a closed ring (Raker et al. 1996; Kambach et al. 1999; Stark et al. 2001; Urlaub et al. 2001). Intriguingly, peptides corresponding to only a subset of these Sm proteins were identified in the purified human telomerase sample.

To test the specificity of association of SmB and SmD3 with human telomerase, we transiently transfected 293T human embryonic kidney cells with expression constructs encoding Sm subunits fused to an N-terminal 
tandem affinity-purification (TAP) tag. TAP-tagged proteins were affinity-purified from whole-cell extracts using IgG agarose, which binds the tandem Protein A domains of the TAP tag. Bound protein complexes were then assayed for enrichment of hTR and telomerase activity. We initially tested a panel of Sm proteins that included two $\mathrm{SmB}$ splice isoforms ( $\mathrm{SmB}$ and $\left.\mathrm{SmB}^{\prime}\right)$, SmD3, and SmG, an Sm protein not detected in the human telomerase purification. As negative and positive controls, we performed parallel affinity purifications using extracts from cells expressing either the TAP tag alone or TAP-tagged dyskerin, a known human telomerase RNP component (Mitchell et al. 1999b).

Immunoblot analysis of whole-cell extracts confirmed that each TAP-tagged protein was properly expressed and accumulated to similar levels (Fig. 1A). RNA from the input lysates and immunopurification-bound fractions was extracted and used for Northern blot analysis (Fig. 1B). Endogenous hTR is detected on denaturing polyacrylamide gels as a doublet of full-length, differentially folded conformers. We detected hTR recovery with the positive control dyskerin, $\mathrm{SmB}, \mathrm{SmB}^{\prime}$, and $\mathrm{SmD} 3$ (Fig. 1B, lanes $8-10,12$ ) but not with the TAP tag alone (Fig. 1B, lane 7,-) or SmG (Fig. 1B, lane 11). By comparison, U2 snRNA, a known RNA partner of Sm proteins, was recovered with each $\mathrm{Sm}$ protein, but not with dyskerin. The TAP tag, therefore, does not block the association of tagged Sm proteins with RNA. We note that tagged SmB

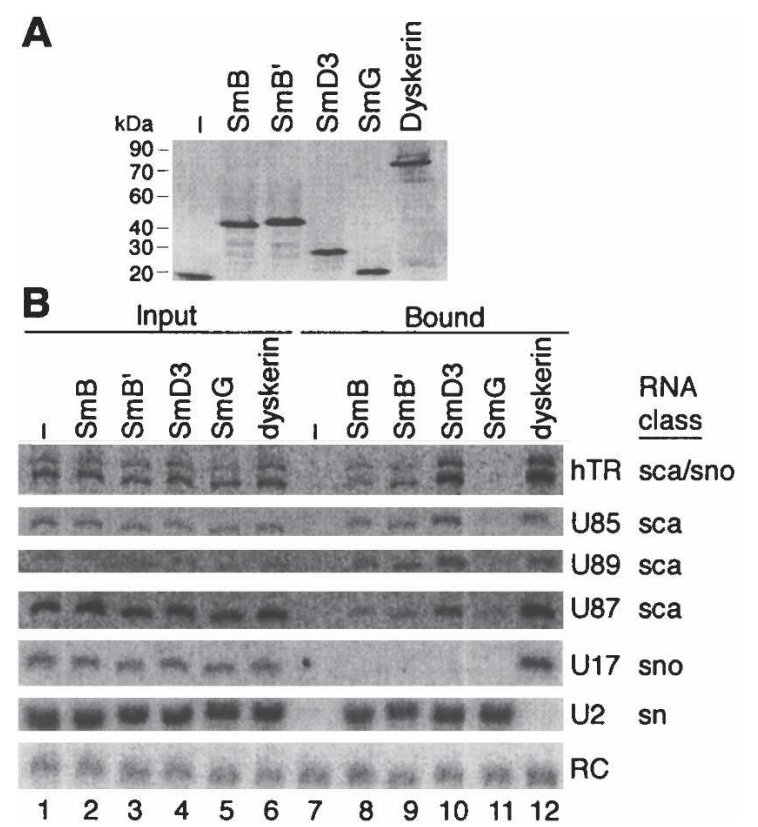

Figure 1. $\mathrm{SmB}$ and $\mathrm{SmD} 3$ copurify human telomerase and Cajal body RNAs. (A) TAP-tagged proteins expressed by transient transfection accumulate to similar levels and are soluble in extract. 293T cells were transiently transfected with constructs expressing the TAP tag alone (-) or the indicated TAP-tagged protein. Whole-cell extracts were analyzed by immunoblot to detect the TAP tag. Protein size standards are indicated to the left in kiloDaltons (kDa). $(B)$ Purification of SmB and SmD3 enriches for hTR and scaRNAs. The TAP tag alone (-) and the TAP-tagged proteins indicated were purified from the transfected $293 \mathrm{~T}$ whole-cell extracts analyzed in $A$. RNA from $5 \%$ of input lysates or $100 \%$ of bound fractions was extracted and analyzed by Northern blot. RC denotes a recovery control added before RNA extraction. copurified a generally lower amount of associated RNAs than did tagged SmD3, which could derive from lessoptimal competition of the N-terminally tagged protein with its endogenous counterpart.

Because mass spectrometry is not quantitative for peptide recovery, it remained possible that $\mathrm{Sm}$ proteins other than SmB and SmD3 would also copurify hTR. Therefore, we expanded the panel of tagged Sm-fold proteins to include each of the prototypical Sm proteins and Lsm10, a subunit of the alternate heptamer found within the U7 snRNP (Pillai et al. 2001). Immunoblot analysis of whole-cell extracts prepared from transiently transfected cells confirmed the proper expression and accumulation of each TAP-tagged protein (Fig. 2A). Moreover, each tagged Sm protein was functional as assessed by its ability to copurify U2 snRNA (Fig. 2B, lanes 9-14). As expected, no U2 snRNA was recovered with Lsm10 or dyskerin (Fig. 2B, lanes 15,16). Surprisingly, only the SmD3 (and SmB) purifications enriched hTR (Figs. 1B [lanes 8-10], 2B [lane 11]) relative to the background level detected with affinity purification of SmD1, SmD2, SmE, SmF, SmG, or Lsm10 (Fig. 2B, lanes 9,10,12-15). We consistently observed lower recovery of U2 snRNA with TAP-tagged SmD1 and SmE, suggesting that the tag on these proteins could interfere with RNP assembly.

To test whether the fully assembled telomerase holoenzyme was associated with $\mathrm{SmB}$ and SmD3, we assayed the purified SmB and SmD3 complexes for telomerase activity. We used the PCR-based telomeric repeat amplification protocol (TRAP) with serial dilutions of the purified samples (Fig. 2C). While the mock purification from extract of cells transfected to express the TAP tag alone yielded only background telomerase activity, the $\mathrm{SmB}$ and SmD3 purifications enriched for active telomerase. Copurification of telomerase activity with $\mathrm{SmB}$ and SmD3 was additionally confirmed by immunopurification of FLAG-tagged versions of the proteins with additional positive and negative controls (Supplementary Fig. 1). Together, the findings described above provide compelling evidence that a subset of the Sm proteins, specifically SmB and SmD3, associate with at least a subpopulation of endogenous human telomerase. We note that human telomerase RNP composition is likely to vary within an individual cell and with cell type, growth conditions, and stages of the cell cycle; some assemblies of telomerase RNP may therefore lack Sm protein interactions.

\section{SmB and SmD3 also associate with scaRNAs}

Human telomerase RNA contains a box H/ACA motif shared with snoRNAs and scaRNAs; it also differs from both canonical snoRNAs and scaRNAs in its possession of one rather than zero or two copies of the CAB box, respectively. Therefore, hTR association with a subset of $\mathrm{Sm}$ proteins could be unique to hTR or it could be a shared feature of H/ACA-motif snoRNAs and/or scaRNAs. We investigated the presence of snoRNAs and scaRNAs in the bound fractions of the Sm-protein affinity purifications described above. Strikingly, every scaRNA tested was specifically enriched in the SmB, $\mathrm{SmB}^{\prime}$, and SmD3 purifications, but not in the purification of any other Sm-fold protein (Figs. 1B [lanes 8-11], 2B [lanes 9-15]; data not shown). This interaction specificity is conspicuously identical to that of hTR. Notably, snoRNAs of either the box H/ACA family (U17 


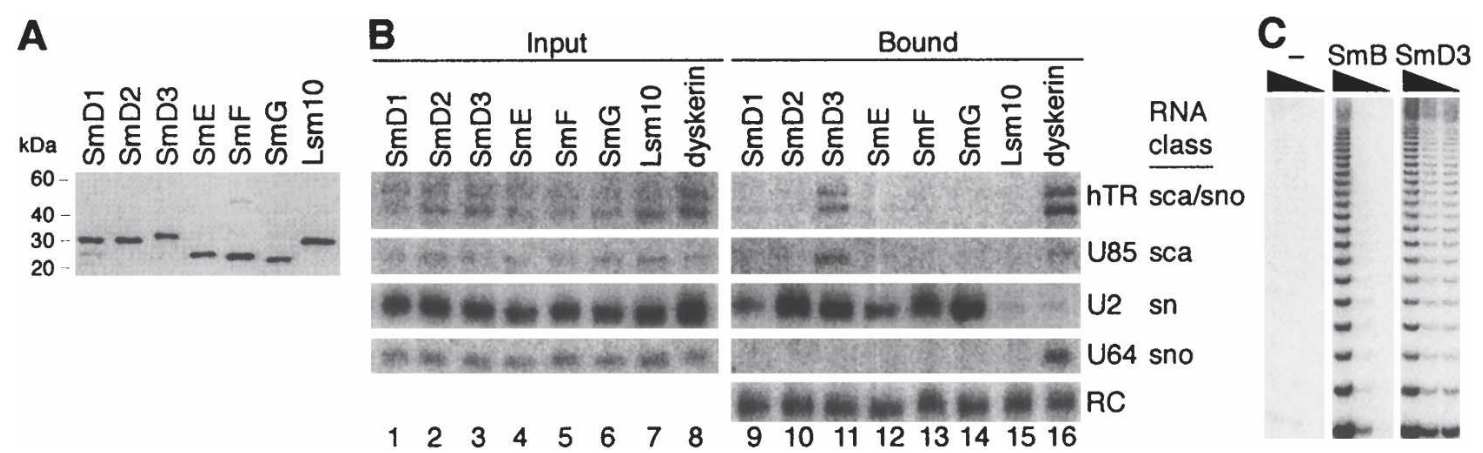

Figure 2. Human telomerase and Cajal body RNAs associate with a subset of Sm-fold proteins. (A) TAP-tagged proteins expressed by transient transfection accumulate to similar levels and are soluble in extract. 293T cells were transiently transfected with constructs expressing the indicated TAP-tagged proteins. Whole-cell extracts were analyzed by immunoblot to detect the TAP tag. Protein size standards are indicated to the left in kiloDaltons $(\mathrm{kDa}) .(B)$ Telomerase and Cajal body RNAs copurify with SmB and SmD3, but not other Sm-fold proteins. The indicated TAP-tagged proteins were purified from the transfected 293 T whole-cell extracts analyzed in A. RNA from $5 \%$ of input lysates or $100 \%$ of bound fractions was extracted and analyzed by Northern blot. (RC) A recovery control added before RNA extraction. (C) Purification of SmB and SmD3 enriches for telomerase holoenzyme. Samples were affinity-purified from extracts of cells expressing the TAP tag alone (-) or TAP-tagged SmB or SmD3. Each bound fraction was used for TRAP assays as a series of fivefold dilutions.

and U64) or the structurally distinct box C/D family (U3) were not significantly enriched in any of the Sm-protein affinity purifications (Figs. 1B [lanes 8-11], 2B [lanes 9-15]; data not shown). Thus, telomerase and Cajal body RNPs are distinguished from other nuclear or nucleolar RNPs in an association with SmB and SmD3 apparently without the complete set of Sm-protein ring subunits.

\section{Endogenous Sm proteins are associated with hTR and scaRNAs}

To further confirm the association of SmB and SmD3 with hTR and scaRNAs, we performed an immunopurification assay using an antibody specific for endogenous $\mathrm{Sm}$ proteins. Sm protein complexes were immunopurified from HeLa human carcinoma cell nuclear extract using the Y12 monoclonal antibody (Lerner et al. 1981), which recognizes similar epitopes on a subset of Sm proteins including, but not restricted to, $\mathrm{SmB}$ and $\mathrm{SmD} 3$ (Brahms et al. 2000). We performed a parallel control immunopurification using a monoclonal antibody specific for Myc. After recovery of antibody complexes, RNA was extracted from the bound fractions and probed by Northern blot (Fig. 3). As expected, Y12 antibody (Fig. 3, lane 3) recovered the U2 snRNA. In contrast, no detectable U2 snRNA was recovered by the control antibody (Fig. 3, lane 2). Importantly, hTR and scaRNAs were also recovered by the Y12 antibody, but not the control antibody. A differential Y12 antibody enrichment of snRNAs relative to scaRNAs is expected due to the difference in number of Y12 epitopes in snRNPs and scaRNPs. The Y12 antibody enrichment of U2 snRNA, hTR, and scaRNAs was specific because there was no such enrichment of the snoRNAs U17 and U64. We repeated this experiment using stringent immunopurification conditions and formaldehyde cross-linked cell extracts with similar results (Supplementary Fig. 2). The association of endogenous Sm proteins with hTR and scaRNAs, but not snoRNAs, is consistent with our tagged protein affinity purification results and verifies endogenous Sm protein association with human telomerase and Cajal body RNPs.

\section{The $C A B$ box determines $S m$ protein interaction with $h T R$}

The prototypical heteroheptameric ring of Sm proteins assembles on specific, U-rich single-stranded RNA sequences (Khusial et al. 2005). Inspection of mammalian scaRNAs revealed no obvious consensus Sm-binding site (data not shown). We therefore suspected that the specificity of association of $\mathrm{SmB}$ and SmD3 with hTR and scaRNAs is distinct from the specificity of association of the canonical Sm ring with its U-rich binding site. Beside the H/ACA motif, hTR and scaRNAs also share the CAB box that mediates association of RNAs with Cajal bodies (Jády et al. 2004). The CAB box has a consensus sequence 5'-UGAG-3', with highest conservation of the final two nucleotides. Alteration of a highly conserved CAB-box nucleotide prevents CAB-box function, with concomitant relocalization of variant RNA from Cajal bodies to nucleoli (Richard et al. 2003; Jády et al. 2004). We specu-

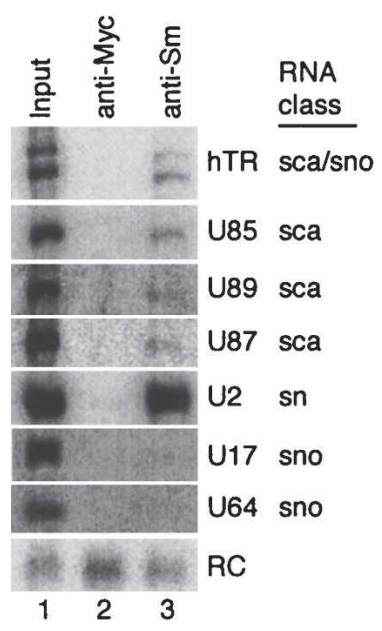

Figure 3. Endogenous Sm proteins copurify hTR and scaRNAs. Immunopurifications were performed from HeLa cell nuclear extract using the monoclonal antibodies anti-Myc 9E10 or anti-Sm Y12. RNA representing $10 \%$ of input or $100 \%$ of bound fractions was analyzed by Northern blot. RC denotes a recovery control added before RNA extraction. 
lated that SmB and SmD3 could associate directly or indirectly with hTR and scaRNAs through their CABbox motif(s). We therefore tested whether alteration of the hTR CAB box would affect the association of Sm proteins with hTR.

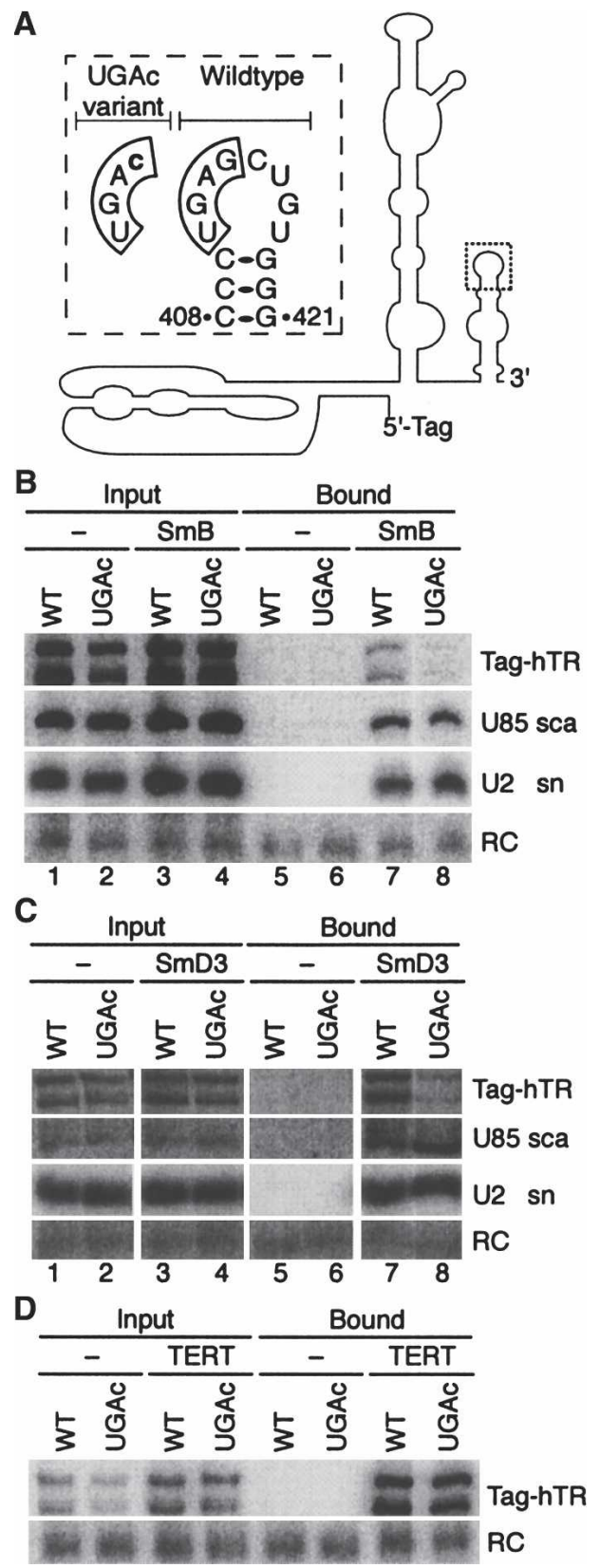

Figure 4. The hTR CAB box is required for association with $\mathrm{SmB}$ and SmD3, but not TERT. $(A)$ The secondary structure of hTR is depicted with an enlargement of the 3 ' stem-loop region indicated by a dashed box. Nucleotides comprising the wild-type CAB box and the UGAc CAB-box variant sequence are indicated within solid outlines. $(B-D)$ Alteration of the hTR CAB box disrupts hTR association with SmB and SmD3, but not TERT. Constructs expressing tagged wild-type hTR (WT) or UGAc hTR CAB-box variant were cotransfected into 293T cells with constructs expressing either the TAP tag alone (-) or a TAP-tagged protein as indicated. Following affinity purification on IgG resin, RNA from $5 \%$ of input lysates or $100 \%$ of bound fractions was analyzed by Northern blot. RC denotes a recovery control added before RNA extraction.
An hTR mutation at the $3^{\prime}$ position of the CAB box altering guanosine to cytidine (the UGAc variant) (Fig. 4A), results in loss of Cajal body localization and enhanced accumulation at nucleoli (Jády et al. 2004). Expression constructs for wild-type and this CAB-box mutant hTR were cotransfected with expression constructs encoding TAP-tagged SmB and SmD3 proteins. Extracts from transfected cells were used for affinity purifications as described above. In order to distinguish recombinant hTR from the endogenous wild-type form, recombinant hTR was extended at the $5^{\prime}$ terminus with a hairpin tag (Tag-hTR). The tag does not affect hTR processing, accumulation, or function (data not shown).

Northern blot analysis indicated that wild-type and UGAc Tag-hTR accumulated to comparable levels as properly processed full-length transcripts (Fig. 4B,C, lanes 1-4). However, wild-type and UGAc Tag-hTR displayed strikingly different levels of association with SmB and SmD3. SmB and SmD3 affinity purifications recovered wild-type Tag-hTR (Fig. 4B,C, lanes 7) well above the background level of binding detected using extracts from cells expressing the TAP tag alone (Fig. 4B,C, lanes $5)$, but the UGAc Tag-hTR variant was not similarly enriched (Fig. 4B,C, lanes 6,8). This difference in SmB and SmD3 copurification of wild-type versus UGAc Tag-hTR was not due to a difference in extract quality, because all Sm protein affinity purifications recovered the endogenous U85 scaRNA and U2 snRNA. These results reveal that the UGAc hTR CAB-box variant is greatly impaired in its association with $\mathrm{SmB}$ and SmD3. In contrast, telomerase reverse transcriptase (TERT) assembly with hTR was not affected by substitution of the CAB box (Fig. 4D). The SmB and SmD3 proteins, either alone or as part of a larger assembly, could recognize the CAB box directly. Alternatively, $\mathrm{SmB}$ and $\mathrm{SmD} 3$ could associate with telomerase and Cajal body RNPs in a different manner, indirectly linked to the $\mathrm{CAB}$ box through a CABbox-dependent event such as Cajal body localization.

\section{A biogenesis-independent function of hTR association with $\mathrm{SmB}$ and $\mathrm{SmD} 3$}

We next examined putative cellular RNP biogenesis roles for the hTR CAB box and hTR assembly with $\mathrm{SmB}$ and SmD3. As noted above, substitution of the second guanosine of the CAB box (the UGAc variant) shifted hTR in situ hybridization signal to nucleoli, while substitution of the first guanosine (the UcAG variant) induced partial relocalization (Jády et al. 2004). These two single-nucleotide variants of hTR and a variant with a tetraloop replacing all eight of the $3^{\prime}$ loop nucleotides (Fu and Collins 2003) were expressed in untagged form by transient transfection of a VA13 human lung fibroblast cell line lacking endogenous hTR and constitutively expressing TERT from an integrated retroviral vector. Substitution of the entire 3 ' loop containing both the $\mathrm{CAB}$ box and an hTR biogenesis signal drastically reduced hTR accumulation as reported previously (Fu and Collins 2003), but substitutions of the CAB box alone did not (Fig. 5A). We also assayed transfected cell extracts for telomerase activity. Consistent with unimpeded TERT assembly (Fig. 4D), the hTR variants with CAB-box substitutions assembled into catalytically active telomerase holoenzyme (Fig. 5B).

Finally, we examined the potential CAB-box dependence of hTR Cajal body transit. Some previous studies 


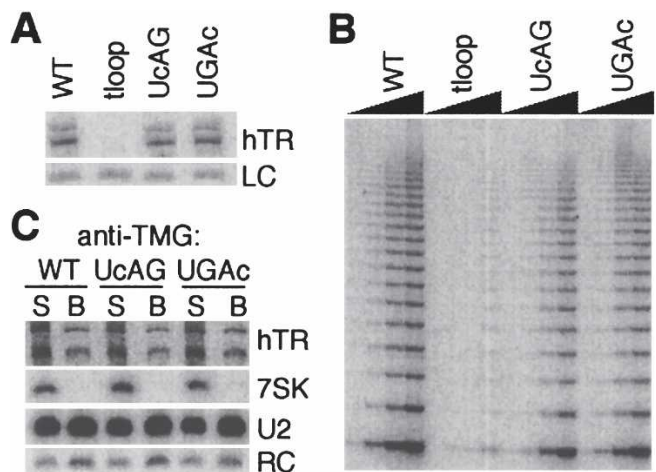

Figure 5. The $\mathrm{CAB}$ box is not required for hTR processing, accumulation, or catalytic activity. A construct encoding untagged wildtype hTR, hTR with a single-nucleotide substitution of the CAB box (UcAG is moderately disruptive; UGAc is severely disruptive), or hTR with the entire $3^{\prime}$ loop substituted by a tetraloop (tloop) was transiently transfected into VA13 cells constitutively expressing TERT. (A) RNA isolated from transfected cell extracts was analyzed by Northern blot. An endogenous RNA that cross-reacts with the hTR probe confirmed equal sample loading (LC). (B) Transfected cell extracts were assayed for telomerase activity by TRAP. Each extract was tested in a series of fivefold dilutions normalized by total protein concentration. $(C)$ Transfected cell total RNA was incubated with anti-TMG antibody. RNA from $25 \%$ of supernatant $(\mathrm{S})$ or $100 \%$ of bound (B) fractions was analyzed by Northern blot. RC denotes a recovery control added before RNA extraction.

have suggested that hTR bears a $5^{\prime}$-trimethylguanosine (TMG) cap (Jády et al. 2004), although other reports have not detected this modification (Lukowiak et al. 2001; Bachand et al. 2002). An hTR TMG cap would likely be added in transit through Cajal bodies (Verheggen et al. 2002). Using a monoclonal antibody that recognizes the TMG cap, we performed immunopurifications from total RNA of VA13 cells expressing untagged wild-type hTR or hTR with either of the single-nucleotide substitutions of the CAB box (Fig. 5C). We detected the positive control TMG-capped U2 snRNA in the bound fraction, while the negative control 7SK RNA containing a monomethyl cap was detected only in the supernatants. The wild-type and variant hTRs were also recovered in the bound fraction, with efficiency independent of CABbox sequence. These results suggest that the hTR CAB box, and the resulting association of $\mathrm{SmB}$ and SmD3, serves a post-biogenesis, potentially regulatory role.

\section{Unique Sm protein association specificity with hTR and scaRNAs}

Sm and Lsm complexes can have different composition and function (Khusial et al. 2005). To date, all of the known Sm and Lsm protein assemblies have a conserved ring-shaped architecture built through interactions of the Sm-fold motifs of each subunit. Our findings reveal a distinguishing Sm protein association specificity of Cajal body resident RNPs. SmB and SmD3 proteins could have sufficient RNA-binding specificity to recognize the CAB box directly, either alone or in association with other Sm-fold proteins. Alternatively, other RNA motifs or protein factors might be involved in establishing the specificity of SmB and SmD3 association with telomerase and Cajal body RNPs.

A requirement for Sm proteins in telomerase RNP biogenesis has been previously described in the budding yeast Saccharomyces cerevisiae. Like an snRNA, yeast telomerase RNA assembles $\mathrm{Sm}$ proteins on its U-rich Sm-binding site (Seto et al. 1999). The results described here establish that hTR association with Sm proteins requires an intact $\mathrm{CAB}$ box rather than a canonical U-rich Sm-binding site. It appears that yeast and vertebrate telomerases independently gained Sm protein associations and acquired different pathways of RNP biogenesis and regulation.

\section{Materials and methods}

Recombinant protein and RNA analyses

The cDNAs encoding proteins described in this study were amplified by RT-PCR from HeLa cell total RNA and cloned into the pcDNA3.1 vector (Invitrogen) for expression as N-terminal TAP tag fusion proteins. The recombinant hTR expression construct was created by inserting two copies of a hairpin that binds the MS2 bacteriophage coat protein between the U3 promoter and mature hTR sequence in the previously described U3-hTR-500 construct (Fu and Collins 2003). Transient transfection and whole-cell extract production were performed as previously described (Mitchell and Collins 2000). Whole-cell extract from transiently transfected cells $(1 \mathrm{mg}$ ) was rotated with $6 \mu \mathrm{L}$ of $\mathrm{IgG}$ resin (Sigma) for $2 \mathrm{~h}$ at $4^{\circ} \mathrm{C}$ in lysis buffer $\left(20 \mathrm{mM}\right.$ HEPES at $\mathrm{pH} 7.9,2 \mathrm{mM} \mathrm{MgCl}_{2}, 0.2 \mathrm{mM}$ EGTA, 10\% glycerol, $1 \mathrm{mM}$ DTT, $0.1 \mathrm{mM}$ phenylmethylsulfonyl fluoride, $0.1 \% \mathrm{NP}-40$ ) with $150 \mathrm{mM} \mathrm{NaCl}$. Resin was then extensively washed using the same buffer. In some cases it was useful to perform additional washes at room temperature with the same buffer containing $0.1 \%$ TX-100. Telomerase activity was analyzed by the modified TRAP assay as previously described (Mitchell et al. 1999a). RNA was extracted using TRIzol (Invitrogen) and analyzed by Northern blot hybridization with oligonucleotide probes complementary to the specified RNA (sequences available upon request). Tag-hTR could be detected with a probe specific for hTR, because Tag-hTR and endogenous hTR have distinct electrophoretic mobilities. Immunoblot analysis used purified rabbit IgG (Sigma) to detect the TAP tag.

Endogenous Sm protein purification

Anti-Myc 9E10 antibody or anti-Sm Y12 antibody was incubated with 1 mg of nuclear extract prepared from HeLa S3 cells. Nuclear extract was diluted with the lysis buffer described above to adjust $\mathrm{NaCl}$ concentration to $\sim 150 \mathrm{mM}$. Following rotation at $4^{\circ} \mathrm{C}$ for $1 \mathrm{~h}$, Protein $\mathrm{G}$ Sepharose was added to the extract with rotation for an additional $1 \mathrm{~h}$. The resin was then washed as described above prior to RNA extraction.

\section{Anti-TMG cap immunopurification}

K121 mouse monoclonal ascites recognizing the TMG cap was prebound to Protein G Sepharose in NET1 buffer (50 mM Tris $\mathrm{HCl}$ at $\mathrm{pH} 7.5,150$ $\mathrm{mM} \mathrm{NaCl}, 0.1 \%$ NP-40, $0.05 \mathrm{mM}$ phenylmethylsulfonyl fluoride). Purified total RNA from transfected cells $(50 \mu \mathrm{g})$ was rotated with the antibody beads for $3 \mathrm{~h}$ at $4^{\circ} \mathrm{C}$ and washed extensively with NET1 buffer before RNA extraction.

\section{Acknowledgments}

We thank Joan Steitz and Don Rio for their gracious gifts of antibodies. We also thank members of the Collins lab for valuable discussion and comments on this manuscript. This work was supported by A.C.S. (RSG0116201) and N.I.H. (HL079585) grants to K.C.

\section{References}

Bachand, F., Boisvert, F.M., Cote, J., Richard, S., and Autexier, C. 2002. The product of the survival of motor neuron (SMN) gene is a human telomerase-associated protein. Mol. Biol. Cell 13: 3192-3202.

Blasco, M.A. 2002. Telomerase beyond telomeres. Nat. Rev. Cancer 2: 627-633.

Brahms, H., Raymackers, J., Union, A., de Keyser, F., Meheus, L., and Luhrmann, R. 2000. The C-terminal RG dipeptide repeats of the spliceosomal Sm proteins D1 and D3 contain symmetrical dimethylarginines, which form a major B-cell epitope for anti-Sm autoanti- 
bodies. J. Biol. Chem. 275: 17122-17129.

Cioce, M. and Lamond, A.I. 2005. Cajal bodies: A long history of discovery. Annu. Rev. Cell Dev. Biol. 21: 105-131.

Darzacq, X., Jády, B.E., Verheggen, C., Kiss, A.M., Bertrand, E., and Kiss, T. 2002. Cajal body-specific small nuclear RNAs: A novel class of 24-O-methylation and pseudouridylation guide RNAs. EMBO J. 21: 2746-2756.

Fu, D. and Collins, K. 2003. Distinct biogenesis pathways for human telomerase RNA and H/ACA small nucleolar RNAs. Mol. Cell 11: $1361-1372$.

Gall, J.G. 2003. The centennial of the Cajal body. Nat. Rev. Mol. Cell Biol. 4: 975-980.

Henras, A.K., Dez, C., and Henry, Y. 2004. RNA structure and function in C/D and H/ACA s(no)RNPs. Curr. Opin. Struct. Biol. 14: 335-343.

Jády, B.E., Darzacq, X., Tucker, K.E., Matera, A.G., Bertrand, E., and Kiss, T. 2003. Modification of Sm small nuclear RNAs occurs in the nucleoplasmic Cajal body following import from the cytoplasm. $E M B O$ J. 22: $1878-1888$

Jády, B.E., Bertrand, E., and Kiss, T. 2004. Human telomerase RNA and box H/ACA scaRNAs share a common Cajal body-specific localization signal. J. Cell Biol. 164: 647-652.

Kambach, C., Walke, S., Young, R., Avis, J.M., de la Fortelle, E., Raker, V.A., Luhrmann, R., Li, J., and Nagai, K. 1999. Crystal structures of two Sm protein complexes and their implications for the assembly of the spliceosomal snRNPs. Cell 96: 375-387.

Khusial, P., Plaag, R., and Zieve, G.W. 2005. LSm proteins form heptameric rings that bind to RNA via repeating motifs. Trends Biochem. Sci. 30: 522-528.

Lerner, E.A., Lerner, M.R., Janeway Jr., C.A., and Steitz, J.A. 1981. Monoclonal antibodies to nucleic acid-containing cellular constituents: Probes for molecular biology and autoimmune disease. Proc. Nat1. Acad. Sci. 78: 2737-2741.

Lukowiak, A.A., Narayanan, A., Li, Z.H., Terns, R.M., and Terns, M.P. 2001. The snoRNA domain of vertebrate telomerase RNA functions to localize the RNA within the nucleus. RNA 7: 1833-1844.

Mitchell, J.R. and Collins, K. 2000. Human telomerase activation requires two independent interactions between telomerase RNA and telomerase reverse transcriptase in vivo and in vitro. Mol. Cell 6: 361-371.

Mitchell, J.R., Cheng, J., and Collins, K. 1999a. A box H/ACA small nucleolar RNA-like domain at the human telomerase RNA 3' end. Mol. Cell. Biol. 19: 567-576.

Mitchell, J.R., Wood, E., and Collins, K. 1999b. A telomerase component is defective in the human disease dyskeratosis congenita. Nature 402: 551-555.

Pederson, T. 2004. Can telomerase be put in its place? J. Cell Biol. 164: 637-639.

Pillai, R.S., Will, C.L., Luhrmann, R., Schumperli, D., and Muller, B. 2001. Purified U7 snRNPs lack the Sm proteins D1 and D2 but contain Lsm10, a new $14 \mathrm{kDa}$ Sm D1-like protein. EMBO J. 20: 54705479 .

Raker, V.A., Plessel, G., and Luhrmann, R. 1996. The snRNP core assembly pathway: Identification of stable core protein heteromeric complexes and an snRNP subcore particle in vitro. EMBO J. 15: 22562269

Richard, P., Darzacq, X., Bertrand, E., Jády, B.E., Verheggen, C., and Kiss, T. 2003. A common sequence motif determines the Cajal body-specific localization of box H/ACA scaRNAs. EMBO J. 22: 4283-4293.

Seto, A.G., Zaug, A.J., Sobel, S.G., Wolin, S.L., and Cech, T.R. 1999. Saccharomyces cerevisiae telomerase is an Sm small nuclear ribonucleoprotein particle. Nature 401: 177-180.

Stark, H., Dube, P., Luhrmann, R., and Kastner, B. 2001. Arrangement of RNA and proteins in the spliceosomal U1 small nuclear ribonucleoprotein particle. Nature 409: 539-542.

Urlaub, H., Raker, V.A., Kostka, S., and Luhrmann, R. 2001. Sm proteinSm site RNA interactions within the inner ring of the spliceosomal snRNP core structure. EMBO T. 20: 187-196.

Verheggen, C., Lafontaine, D.L., Samarsky, D., Mouaikel, J., Blanchard, J.M., Bordonne, R., and Bertrand, E. 2002. Mammalian and yeast U3 snoRNPs are matured in specific and related nuclear compartments. EMBO J. 21: 2736-2745

Yu, Y.T., Shu, M.D., and Steitz, J.A. 1998. Modifications of U2 snRNA are required for snRNP assembly and pre-mRNA splicing. EMBO J.
17: 5783-5795.

Zhu, Y., Tomlinson, R.L., Lukowiak, A.A., Terns, R.M., and Terns, M.P. 2004. Telomerase RNA accumulates in Cajal bodies in human cancer cells. Mol. Biol. Cell 15: 81-90. 


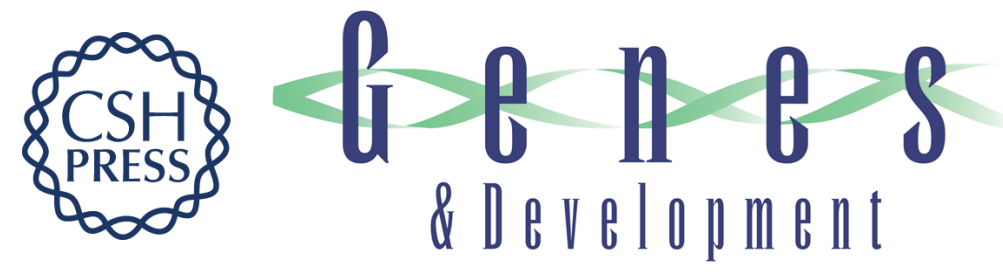

\section{Human telomerase and Cajal body ribonucleoproteins share a unique specificity of Sm protein association}

Dragony Fu and Kathleen Collins

Genes Dev. 2006, 20:

Access the most recent version at doi:10.1101/gad.1390306

Supplemental
Material http://genesdev.cshlp.org/content/suppl/2006/02/15/gad.1390306.DC1

References This article cites 27 articles, 14 of which can be accessed free at: http://genesdev.cshlp.org/content/20/5/531.full.html\#ref-list-1

License

Email Alerting

Receive free email alerts when new articles cite this article - sign up in the box at the top

Service

right corner of the article or click here.

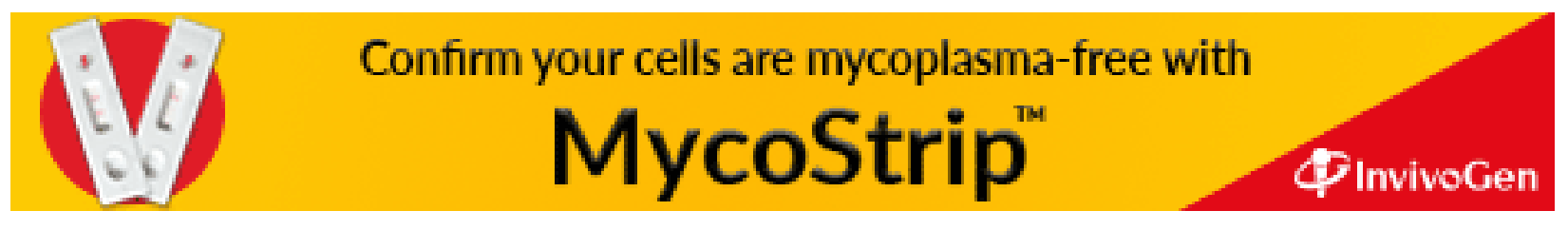

